\title{
Successful treatment with tumor necrosis factor- $\alpha$ blockers for poison-induced liver injury: case report and literature review
}

\author{
Qian Xing $^{1,2} \wedge$, Biao Zhu ${ }^{1,2}$ \\ ${ }^{1}$ Department of Anaesthesia, Critical Care and Pain Medicine, Fudan University Shanghai Cancer Center, Shanghai, China; ${ }^{2}$ Department of \\ Oncology, Shanghai Medical College, Fudan University, Shanghai, China \\ Correspondence to: Biao Zhu. ICU, Fudan University Shanghai Cancer Center, No. 270 Dongan Road, Shanghai 200032, China. \\ Email: zhubiaozs@sohu.com.
}

\begin{abstract}
Acute poisoning could result in hepatic dysfunction which is potentially life threatening. We reviewed three cases of poison-induced liver injury with gastrointestinal disorder on admission. Two cases were poisoned by mushroom $\alpha$-Amanitin while the other was poisoned by acetaminophen (APAP). They were cured under the close monitor of laboratory examinations and other supportive therapies, as well as the off-label medication of etanercept, a kind of tumor necrosis factor- $\alpha$ (TNF- $\alpha$ ) blockers with written informed consent. Among them, casel was given the first dose doubling of TNF- $\alpha$ blockers for higher liver enzyme levels. There is a lack of effective and safe treatments for poison-induced liver injury. TNF- $\alpha$ has been proved to play an important role in the aggravation of liver injury and the start-up of inflammatory cascade reaction. Therapy with TNF- $\alpha$ blockers shown potential therapeutic efficacy in hepatic dysfunction by some researches. Anyway, no strong recommendation could be drawn from these small sample size studies. On the other side, TNF- $\alpha$ could also mediate an opposing effect for hepatocytes since the hepatic toxicity of TNF- $\alpha$ blockers has generated attentions. The safety for the off-label medication of TNF- $\alpha$ blockers in liver injury, however, still lacks strong evidences. More experimental and clinical researches are needed to focus on potential mechanisms.
\end{abstract}

Keywords: Case report; liver injury; tumor necrosis factor- $\alpha$ (TNF- $\alpha$ ); Etanercept

Submitted Jun 19, 2020. Accepted for publication Nov 06, 2020.

doi: 10.21037/apm-20-1282

View this article at: http://dx.doi.org/10.21037/apm-20-1282

\section{Introduction}

Acute poisoning can cause acute liver injury (ALI) and even acute liver failure (ALF). However, effective and safe treatments are lacking to control the progressive deterioration of liver function after acute poisoning (1). Some researchers have indicated that tumor necrosis factor- $\alpha(T N F-\alpha)$ may play a pivotal role in the process of ALF by participating in the inflammatory cascade and subsequently impairing the visceral function of the liver (2). This paper presents three cases in which poison-induced liver injury was successfully treated with TNF- $\alpha$ blockers (recombinant human TNF- $\alpha$ receptor II: IgG Fc fusion protein, etanercept). We present the following case report in accordance with the CARE reporting checklist (available at http://dx.doi.org/10.21037/apm-20-1282).

\section{Case presentation}

All procedures in this research that were performed on human participants were conducted in accordance with the ethical standards of the institutional and the Declaration of Helsinki (as revised in 2013). Written informed consent was obtained from all participants.

Case 1, a previously healthy, 34-year-old Chinese

^ ORCID: 0000-0002-2016-8930. 


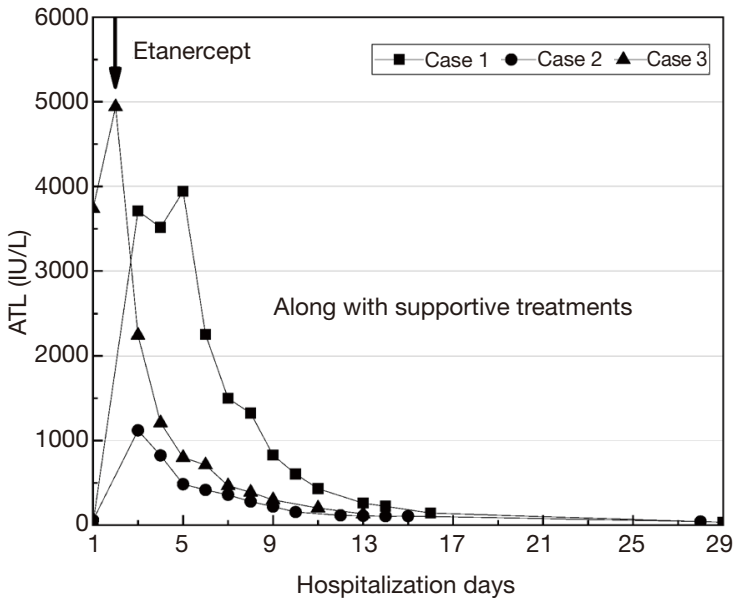

Figure 1 The variation tendency of alt of the three cases. ALT, alanine aminotransferase, normal range, 0-75 IU/L.

female was admitted to the emergency department (ED) with the symptoms of refractory nausea, vomiting, watery diarrhea, and cold sweats, which had lasted for 3 days after eating wild mushrooms. She did not present with fever, hematemesis, syncope, or disturbance of consciousness. The patient's blood pressure was $80 / 60 \mathrm{mmHg}$ at the time of referral to the ED, with other vital signs and physical examination results appearing benign overall. The leftover samples of the mushrooms she had ingested were sent to the Shanghai Academy of Agricultural Sciences, China, and identified through gross morphology to be Lepiota brunneoincarnata Chod. et Mart. (Genus: Lepiota, Species: L. brunneoincarnata). The patient's lab tests showed a gradual increase in the indices of alanine aminotransferase (ALT), aspartate transaminase, total bilirubin, and prothrombin time. Considering her progressive and aggravated clinical symptoms and laboratory parameters, the patient was diagnosed with acute mushroom intoxication and was considered to be at short-term risk of ALF; emergent hemodialysis (HD) was prepared for treatment. According to the recommendations of a consultant from the Digestive Department (DD), the patient received treatment with 3 etanercept injections $(50 \mathrm{mg}$ on day 2 , and $25 \mathrm{mg}$ on days 8 and 11 of hospitalization), as well as traditional supportive treatment including methylprednisolone, $\gamma$-immunoglobulin, reduced glutathione (GSH), polyene phospholipids, and coenzyme complex. On day 5 of admission, the patient's ALT level began to decrease rapidly, and her clinical manifestations improved. She was discharged on day 16 with almost normal laboratory results.
An outpatient follow-up visit 2 weeks later showed that the patient's liver enzyme measurements had fully recovered (Figure 1).

Case 2 was a 50-year-old Chinese male without prior known disease. And he was the husband of the patient from case 1 . He had ingested the same mushrooms and displayed exactly the same gastrointestinal symptoms as his wife. On admission, there were no abnormalities in his vital signs or physical examination results. From his lab results, the patient was determined to have a lower peak value of ALT than his wife. Based on his condition, he was given an injection of $25 \mathrm{mg}$ etanercept on days 3, 6, and 9 of hospitalization, coupled with supportive treatment similar to that of his wife. He was discharged on day 15 , as the level of ALT was approximately normal. He had completely recovered 2 weeks later (Figure 1).

Case 3 was a healthy 25 -year-old female who had been ill for 2 days following purposeful consumption of $>20$ tablets of $\mathrm{N}$-acetyl-para-aminophenol (APAP) after a quarrel with her family. She was referred to the ED with symptoms including nausea, vomiting, and dizziness. Her vital signs and physical examination were normal on admission. APAP was detected in the patient's blood at a concentration of $0.9 \mathrm{ug} / \mathrm{mL}$ by the Shanghai Association of Forensic Science, China. Therefore, she was diagnosed with acute APAP poisoning without indications for emergent HD. Upon recommendations of the consultant from the $\mathrm{DD}$, the patient received 3 etanercept injections $(50 \mathrm{mg}$ on day 2 , and $25 \mathrm{mg}$ on days 5 and 8 of hospitalization) along with supportive treatment similar to that of cases 1 and 2. On day 13 after admission, her laboratory results were almost normal, and she was discharged from the hospital (Figure 1).

\section{Discussion}

Cases 1 and 2 were poisoned by $\alpha$-Amanitin, which is known to be contained in Lepiota brunneoincarnata Chod et Mart. TNF- $\alpha$ is known to aggravate $\alpha$-Amanitin's hepatotoxicity through interacting with its reactive oxygen species (ROS) and shortening its latency period of hepatotoxicity (3). The toxicity mechanism of case 3 was hepatotoxicity by a reactive metabolite of APAP. Treatments for poison-induced liver injury include gastric decontamination, extracorporeal treatment, drug therapy, and in severe cases, eventual liver transplantation (LT). The death rate remains high, despite this range of treatment options (3).

Acute hepatocyte injury brings about mitochondrial injury and the release of multiple damage-associated 
molecular patterns, including high-mobility group box 1 protein (HMGB1). HMGB1 subsequently binds to tolllike receptor 4 (TLR4) on Kupffer cells (KCs) and activates the nuclear factor kappa-light-chain-enhancer of activated $\mathrm{B}$ cells $(\mathrm{NF}-\mathrm{\kappa B})$ signal transduction pathway. This leads to the activation of KCs and secretion of pro-inflammatory cytokines and ROS, resulting in the apoptosis and necrosis of hepatocytes $(4,5)$. TNF- $\alpha$ functions as a homotrimer that binds to $\mathrm{p} 55 \mathrm{TNF}$ receptors (TNF-R1) and p75 TNF receptors (TNF-R2). TNF- $\alpha$ secreted by KCs is the key to the cytokine net and is closely related to ALF aggravation $(5,6)$. First of all, TNF- $\alpha$ can trigger the apoptosis program by promoting TNF-R1 on hepatocytes to express TNF receptor-associated Fas-associated death domain (FADD), and activating the effector caspase-3. Meanwhile, TNF- $\alpha$ gives rise to hepatic hypoxic ischemic injury and destroys hepatic microcirculation. TNF- $\alpha$ upregulates the expression of intercellular and vascular cell adhesion molecule 1 (VCAM-1), causing the swelling and activation of endothelial cells, aggregation of sinusoidal platelets, and invasion of peripheral immune cells. Above all, several downstream transcriptional factors, including $\mathrm{NF}-\kappa \mathrm{B}$, are activated by TNF- $\alpha$, which contributes to the activation of KCs and neutrophil aggregation. Infiltrated neutrophils release ROS and proteases, leading to the apoptosis and necrosis of hepatocytes, which starts the inflammatory cascade reaction.

Over the last decades, TNF- $\alpha$ blockers, including etanercept, infliximab, adalimumab, certolizumab pegol, and golimumab, have been approved for the treatment of autoimmune diseases including inflammatory bowel disease, rheumatologic, and dermatological disorders. Also, it has been reported that TNF- $\alpha$ blockers can be used for treating severe stages of hepatic diseases such as nonalcoholic fatty liver disease, alcoholic hepatitis, autoimmune hepatitis, and primary biliary cholangitis (7). Sun et al. suggested that TNF- $\alpha$ blocker therapy may down-regulate the level of pro-inflammatory cytokines and up-regulate anti-inflammatory cytokines (8). Guo et al. showed that TNF- $\alpha$ blockers could protect against lipopolysaccharide (LPS)-induced ALI by inhibiting the bioactivity of TNF- $\alpha$ and enhancing anti-oxidation (9), while a Canadian study on azoxymethane-induced ALF indicated that systemic block of TNF- $\alpha$ attenuated inflammation and delayed the progression of ALF, which could provide a novel therapeutic approach for patients awaiting LT (2). Further, a Chinese randomized controlled trial on rats with APAP-induced ALI concluded that TNF- $\alpha$ blockers could ameliorate APAP- induced hepatic damage (10). Xu et al. found that TNF- $\alpha$ blockers were considered to have a potential therapeutic value in acute-on-chronic liver failure (ACLF) through improving the survival rate of rats with ACLF induced by D-galactosamine and LPS (11).

In contrast, the hepatic toxicity of TNF- $\alpha$ blockers has drawn much attention, and the number of cases of liver injury, including life-threatening cases, has been reported to be increasing (12). Indeed, studies indicated TNF- $\alpha$ could mediate a dual and opposing effect by acting on TNF-R1 and TNF-R2, promoting both hepatocyte necrosis in response to toxins and conversely increasing liver cell proliferation in appropriate conditions (7). As previously discussed, TNF- $\alpha$ can drive the inflammatory response through TNF-R1 on effector T cells. Moreover, the activation of TNF-R2 expressed on regulatory T cells leads to their expansion and, consequently, to the prevention of autoimmunity and attenuation of inflammation. Therefore, the blockade of TNF- $\alpha$ may determine either further liver injury or regeneration by modulating the balance between effector and regulatory $\mathrm{T}$ cells.

\section{Conclusions}

Here, we have presented three cases of poison-induced liver injury that were successfully treated with TNF- $\alpha$ blockers. Among them, case 1 was given double the standard first dose of etanercept to address her higher liver enzyme levels. However, the safety for the off-label medication of TNF- $\alpha$ blockers in liver injury still lacks strong evidence, since TNF- $\alpha$ blockers could mediate a dual and opposing effect on hepatocytes. Thus, further experimental and clinical research is required to investigate the potential mechanisms.

\section{Acknowledgments}

Funding: None.

\section{Footnote}

Reporting Checklist: The authors have completed the CARE reporting checklist. Available at http://dx.doi.org/10.21037/ apm-20-1282

Conflicts of Interest: Both authors have completed the ICMJE uniform disclosure form (available at http://dx.doi. org/10.21037/apm-20-1282). The authors have no conflicts of interest to declare. 
Ethical Statement: The authors are accountable for all aspects of the work in ensuring that questions related to the accuracy or integrity of any part of the work are appropriately investigated and resolved. All procedures performed in studies involving human participants were in accordance with the ethical standards of the institutional and/or national research committee(s) and with the Helsinki Declaration (as revised in 2013). Written informed consent was obtained from the patients.

Open Access Statement: This is an Open Access article distributed in accordance with the Creative Commons Attribution-NonCommercial-NoDerivs 4.0 International License (CC BY-NC-ND 4.0), which permits the noncommercial replication and distribution of the article with the strict proviso that no changes or edits are made and the original work is properly cited (including links to both the formal publication through the relevant DOI and the license). See: https://creativecommons.org/licenses/by-nc-nd/4.0/.

\section{References}

1. Squires JE, McKiernan P, Squires RH. Acute liver failure: An update. Clin Liver Dis 2018;22:773-805.

2 Chastre A, Belanger M, Beauchesne E, at al. Inflammatory cascades driven by tumor necrosis factor-alpha play a major role in the progression of acute liver failure and its neurological complications. PloS One 2012;7:e49670.

3 Garcia J, Costa VM, Carvalho A, et al. Amanita phalloides poisoning: Mechanisms of toxicity and treatment. Food Chem Toxicol 2015;86:41-55.

4 Woolbright BL, Jaeschke H. Role of the inflammasome in acetaminophen-induced liver injury and acute liver failure. J Hepatol 2017;66:836-48.

5 Zimmermann HW, Trautwein C, Tacke F. Functional role of monocytes and macrophages for the inflammatory response in acute liver injury. Front Physiol 2012;3:56.

6 Dong Y, Liu Y, Kou X, et al. The protective or damaging effect of Tumor necrosis factor- $\alpha$ in acute liver injury is concentration-dependent. Cell Biosci 2016;6:8.

7 Lopetuso LR, Mocci G, Marzo M, et al. Harmful Effects and Potential Benefits of Anti-Tumor Necrosis Factor (TNF)- $\alpha$ on the Liver. Int J Mol Sci 2018;19:2199.

8 Sun XY, Su Y, Ren LM, et al. Effect of a recombinant human tumor necrosis factor receptor-Fc fusion protein on pro-and anti-inflammatory cytokines in rheumatoid arthritis. Chinese Journal of Rheummatology 2016;10:209-12.

9 Guo ZY, Wang ST, Xu MH, et al. Effects of recombinant human TNFR: Fc fusion protein on rat acute liver injury induced by lipopolysaccharide. Chinese Journal of Emergency Medicine 2009;18:1178-82.

10 Zhang L, Zhan J, Wu BH, et al. The role of TNF- $\alpha$ in acetaminophen-induced liver injury in rats. Chinese Journal of Gastroenterology and Hepatology 2014;23:1181-3.

$11 \mathrm{Xu} \mathrm{Y,} \mathrm{Wang} \mathrm{H,} \mathrm{Bao} \mathrm{S,} \mathrm{et} \mathrm{al.} \mathrm{Amelioration} \mathrm{of} \mathrm{liver} \mathrm{injury}$ by continuously targeted intervention against TNFRp 55 in rats with acute-on-chronic liver failure. PLoS One 2013;8:e68757.

12 Kok B, Lester ELW, Lee WM, et al. Acute Liver Failure from Tumor Necrosis Factor- $\alpha$ Antagonists: Report of Four Cases and Literature Review. Dig Dis Sci 2018;63:1654-66.
Cite this article as: Xing Q, Zhu B. Successful treatment with tumor necrosis factor- $\alpha$ blockers for poison-induced liver injury: case report and literature review. Ann Palliat Med 2021;10(6):7042-7045. doi: 10.21037/apm-20-1282 